\title{
Redox Regulation of Cysteine-Dependent Enzymes in Neurodegeneration
}

\author{
Rodney P. Guttmann and Tamara J. Powell \\ Center on Aging, School of Psychological and Behavioral Sciences, University of West Florida, 11000 University Parkway, Pensacola, \\ FL 32514, USA \\ Correspondence should be addressed to Rodney P. Guttmann, rguttmann@uwf.edu
}

Received 21 March 2012; Accepted 10 May 2012

Academic Editor: Pier Giorgio Mastroberardino

Copyright ( 2012 R. P. Guttmann and T. J. Powell. This is an open access article distributed under the Creative Commons Attribution License, which permits unrestricted use, distribution, and reproduction in any medium, provided the original work is properly cited.

Evidence of increased oxidative stress has been found in various neurodegenerative diseases and conditions. While it is unclear whether oxidative stress is a cause or effect, protein, lipid, and DNA have all been found to be susceptible to oxidant-induced modifications that alter their function. Results of clinical trials based on the oxidative-stress theory have been mixed, though data continues to indicate that prevention of high levels of oxidative stress is beneficial for health and increases longevity. Due to the highly reactive nature of the sulfhydryl group, the focus of this paper is on the impact of oxidative stress on cysteine-dependent enzymes and how oxidative stress may contribute to neurological dysfunction through this selected group of proteins.

\section{Introduction}

It is clear that while oxygen is essential for life in order to produce chemical energy in the form of ATP, paradoxically, the byproduct of its metabolism generates multiple reactive oxygen species (ROS) that are associated with cellular toxicity. Specifically, in regards to neurodegeneration, there is substantial evidence that ROS are a major component of diseases including Alzheimer's, Parkinson's, and amyotrophic lateral sclerosis [1-4]. While clinical trials aimed at decreasing the burden of oxidative stress have not clearly demonstrated effectiveness, genetic research has found that high levels of antioxidant enzymes prolong life and decrease pathology. In addition, animal models have also indicated that oxidative stress is an important and consistent characteristic of many forms of neurodegeneration.

One particular group of proteins that appear to be intimately involved in the neurodegenerative processes is the cysteine-dependent proteins. This group includes various proteases, antioxidant enzymes, kinases, phosphatases, and other types of enzymes as well as other nonenzymatic proteins such as those that use cysteine as a structural component rather than as part of a catalytic site. More research will be needed to firmly establish the extent to which oxidative stress is causal in these diseases, but based on current understanding, therapies to reverse the oxidant-induced modifications of proteins, lipids or, DNA are expected to be beneficial. This paper will highlight some selected, yet significant cysteine-dependent enzymatic systems that rely on a proper redox environment for their activity and provide evidence for their redox control in neurodegenerative disease. Potential relationships to cancers will also be discussed.

\section{Redox Sensitivity of Cysteine}

The aminoacid cysteine is highly sensitive to redox state. This is largely due to the reactivity of anionic sulfur to various oxidizing agents that can form multiple types of oxidized species (see Figure 1). However, not all cysteines are equally sensitive, and such sensitivity has been utilized throughout evolution to provide protection against oxidative stress.

A close examination of the variety of physiologically occurring antioxidant systems, that use cysteine as a major component of their antioxidant activity or as part of a redox "sensor," clearly demonstrates the sensitivity and evolutionary significance of cysteine as part of a protein's 

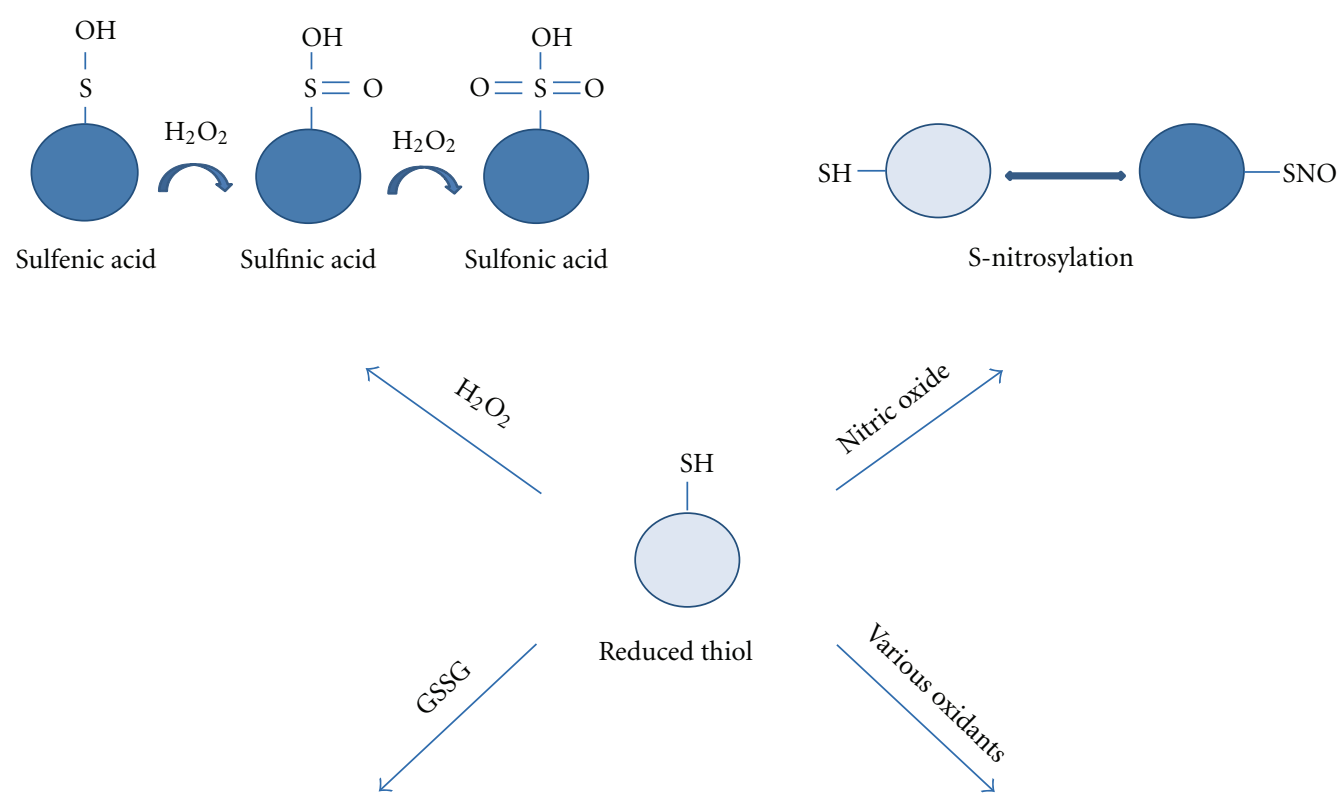

Reduced thiol
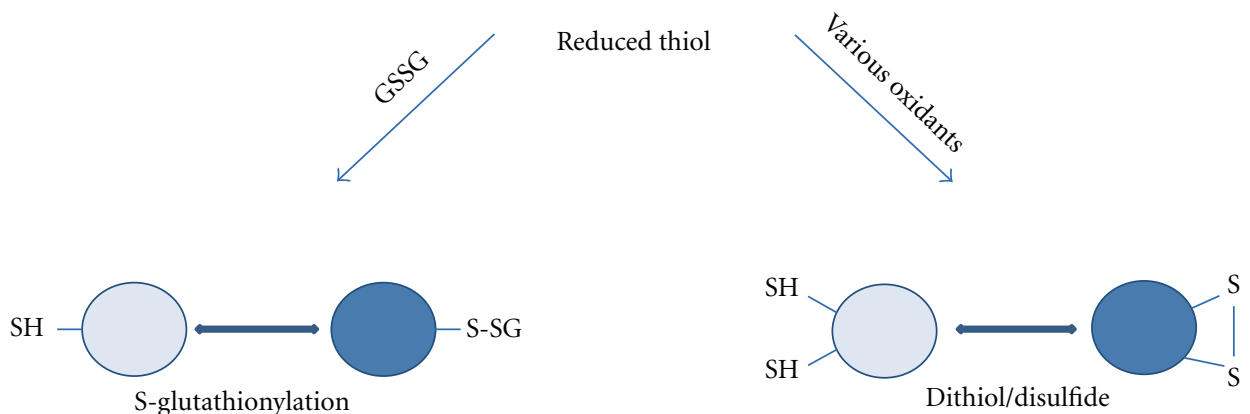

Figure 1: Diagrammatic representation of major oxidation states of cysteine that have been found in vivo. Circles represent a protein that contains a cysteine within its primary structure. In its most reduced state, the sulfur group of cysteine is found in the form of -SH. The sulfur can become modified in a number of ways including S-nitrosylated by nitric oxide or S-glutathionylated by glutathione, which are being increasingly recognized for their importance in regulated many cysteine-containing enzymes. In addition, the sulfur group can be oxidized to sulfenic, sulfinic, and sulfonic acids or it may form an intra- or inter-molecular disulfide bond.

active center [5]. For example, glutathione (GSH) consists of glutamate, glycine, and cysteine and is the major antioxidant found in brain. It is found at millimolar levels and is a major determinant of intracellular redox conditions. Cysteine itself has been shown to be the major extracellular antioxidant. Further examples of cysteines' critical role in redox balance can be found in other enzymatic systems including the multiple enzymes involved in the maintenance of peroxiredoxins, glutaredoxins, and thioredoxins among others. The natural role of cysteines as redox sensors is further observed by the observation that throughout evolution, cysteines are found in transcriptional regulators that are modulated by oxidative stress such as oxyR and Nrf2/Keap [6].

Due to the varying microenvironments that exist for cysteine within a given protein structure, cysteines are not equally reactive. For example, as discussed further below, the Parkinson's disease-linked protein, DJ-1 cysteine at position 106, appears to be highly sensitive to oxidative attack, while two other cysteines within its structure are not as easily modified [7]. Such apparent specificity of cysteines within the same protein is also observed among many other proteins $[8,9]$. In terms of the macroenvironment, cysteinedependent enzymes require a reducing environment for activity, which is the condition maintained in the cytoplasm in contrast to the extracellular space that is oxidizing. However, the lysosomal compartment is variable, and changes in redox state have been shown to modulate enzymatic activities located within it. For example, cathepsin activity was found to be altered through redox state as detected by a change in cleavage pattern produced under varying redox conditions [10]. Thus, the location of the cysteine within the overall tertiary/quaternary structure as well as its macroenvironment (e.g., intracellular versus extracellular or organelle) plays major roles in the extent to which a cysteine is stabilized in the anionic transition state, thereby affecting its reactivity to a change in the redox state.

\section{Sources of Oxidants in Brain}

Environmental toxins are thought to be a significant contributor to neuronal-related disorders including $\mathrm{AD}$ and particularly PD. These include a variety of naturally occurring and synthetic compounds, which results in the production of reactive species through well-characterized chemical pathways including the Fenton reaction and others [11]. In addition to direct chemical means, many of these environmental molecules, such as rotenone or paraquat, target mitochondria and disrupt the efficient production 
of energy, leading to abnormal increases in free radical production such as superoxide [12]. The identification of these toxins and their mechanisms of action is the subject of extensive research with a major emphasis on how their toxicity relates to the production of free radicals.

Besides environmental toxins, there are also important cellular sources of oxidants localized within cytosolic and mitochondrial compartments. Cellular sources include NADPH oxidases, which are enzymes associated with both signal transduction and the killing of foreign organisms through the production of superoxide. Monoamine oxidase (MAO) located at the mitochondrial surface is also a source of hydrogen peroxide. Due to MAO-B's role in the metabolism of dopamine, MAO activity is linked to PD in part due to the production of reactive oxygen species resulting from MAO-mediated metabolism of dopamine [13].

Endogenous superoxide production is strongly associated with the mitochondria and can occur within the matrix, the intermembrane space, and at the outer membrane of mitochondria. For example, reactive species are formed as part of electron transport including complex I (NADHubiquinone oxidoreductase). Complex $\mathrm{I}$ is considered to be an important source of free radical generation $[14,15]$ and does so during either forward electron flow or reverse electron transport [16]. Though debate exists about the mechanisms involved (one-site versus two-site model), the importance of superoxide and hydrogen peroxide formation through the various mitochondrial pathways should not be underestimated as oxidants produced through the mitochondria are considered highly relevant to aging and neurodegeneration.

\section{Oxidative Stress in Neurodegenerative Disease}

Over the last few decades it has become increasingly clear that the human brain is more sensitive to various forms of oxidative attack damage compared to other organs in the body. This is due in large part to the high metabolic activity found in brain and the seemingly limited capacity for the repair of damage to neurons as a result of injury. Many types of oxidizing molecules have been observed in the human brain, and their presence is associated with selective damage to brain regions linked with neurodegenerative disease. While it is uncertain as to the extent in which the increase in reactive species causes the visible pathological hallmarks, the formation of reactive oxygen, nitrogen, or sulfur species is nevertheless generally recapitulated in animal models of each disease, strongly suggesting a potential causal link. Observed biomarkers of increased oxidative stress include 4-hydroxynonenal, thiobarbituric acid-reactive substances, free fatty acid release, and acrolein formation for lipid peroxidation; 8-hydroxy-2-deoxyguanosine for DNA; protein carbonyls, 3-nitrotyrosine, and glutathionylation for proteins. In regards to proteins, along with cysteine, multiple aminoacids are found to be modified including lysine, methionine, histidine, and others.
4.1. Alzheimer's Disease. $\mathrm{AD}$ is an age-associated progressive neurodegenerative disease that affects behavior, cognition, and memory and is characterized by two major pathological hallmarks: extracellular plaques composed primarily of $\mathrm{A} \beta$ and intracellular inclusions of tau protein known as tangles. It currently has no known cause or cure and remains the most common form of irreversible dementia affecting approximately 20 million people worldwide. Oxidative damage is one of the earliest detectable changes observed in both genetic and sporadic forms of Alzheimer's disease [17].

While there are several theories about the source of the various oxidizing molecules, $\mathrm{A} \beta$ has been a prime candidate. Indeed, treatment of various model systems with different $\mathrm{A} \beta$ forms typically results in increased oxidative stress. Recent work has shown that extracellular $\mathrm{A} \beta$ treatment results in atypical redox effects in astrocytes compared to treatment with other oxidizing molecules, suggesting that $\mathrm{A} \beta$ possesses unique oxidizing properties [18]. In addition to the potential for $\mathrm{A} \beta$ to stimulate increased oxidative stress, there is also evidence that major antioxidant systems such as superoxide dismutase, catalase, and others have decreased activity associated with $\mathrm{AD}$ progression [19].

4.1.1. Examples of Oxidized Enzymes in AD. Peroxiredoxins (Prxs) are a family of peroxidases that reduce peroxynitrite and a variety of other hydroperoxides. They use a redoxsensitive cysteines within their active site reducing the peroxide substrates either through the formation of an intramolecular disulfide bond or oxidation to sulfinic acid or sulfonic acid [20]. Proteomic studies for subjects with early $\mathrm{AD}$ found that Prx-2 was oxidized in a brain region containing significant AD-related pathology compared to age-matched controls [21]. In another study by Cumming and colleagues [22], it was not only shown that Prx-2 was more oxidized in $\mathrm{AD}$ brains, but also treatment of cultured primary neurons with $\mathrm{A} \beta$ resulted in Prx oxidation that was reversible by addition of a cysteine-specific antioxidant, $\mathrm{N}$ acetylcysteine. In addition, Fang and colleagues found that Prx-2 was S-nitrosylated at active-site cysteines Cys 51 and Cys 172 [23].

Protein disulfide isomerase (PDI) is a multifunctional enzyme with several family members. These enzymes include chaperone activity mediated by catalyzing the reduction, oxidation, and isomerization of protein disulfides to maintain proper protein folding. PDI redox activity is based on the presence of two thioredoxin-like motifs (CXXC) (human PDI: Cys 36/39 and Cys380/383). It has been found to be oxidized in $\mathrm{AD}$ and colocalizes with neurofibrillary tangles [24]. Though no changes in the amounts of PDI have been noted in AD brain, Uehara and colleagues [25] did show that PDI was S-nitrosylated at multiple cysteines in AD brain and that such oxidation resulted in enzyme inactivation. Since PDI is important for the folding of proteins by catalyzing cysteine-disulfide exchange, its inactivation increased the levels of misfolded of proteins, leading to the activation of the unfolded protein response.

Calpains are calcium and cysteine-dependent endoproteases whose active-sites are sensitive to oxidative inactivation. In addition to $\mathrm{AD}$, calpains play a role in multiple 
disease states, including cancer [26, 27]. As a putative physiological regulator of key proteins associated with $\mathrm{AD}$ such as amyloid precursor protein and tau among others, understanding calpains' potential dysregulation by redox status is important. Calpain's active site cysteine (Cys105) was found to be oxidized, in vitro and in cultured cells only in the presence of calcium $[28,29]$. Presumably, this is because the active site is otherwise inaccessible to oxidative attack when the enzyme is in an inactive conformation. Evidence suggests that calpain-like enzymatic activity is also inhibited in brain regions of AD associated with high pathology [30].

4.2. Parkinson's Disease. Parkinson's disease is the second most common neurodegenerative disease characterized by loss of dopaminergic neurons, glutathione depletion, oxidative stress, and the formation of intracellular inclusions of alpha-synuclein called Lewy bodies. Similar to AD, the vast majority of PD cases are sporadic with only $5-10 \%$ of cases due to genetic causes [31]. Much of what we understand has been gained through the use of animal models of PD that involve the administration of exogenous compounds such as 1-methyl-4-phenyl-1,2,3,6-tetrahydropyridine (MPTP), rotenone, and paraquat. Investigation of these compounds has strongly linked them to mitochondrial dysfunction and the abnormal production of free radicals, which generally reflects what is observed in the human disease. In the course of these studies, several highly relevant cysteine-dependent enzymes known to contribute to PD have been observed to be modified at key cysteine residues by these reactive species.

As discussed above, PDI has also been found to be oxidized in samples of PD brain [25]. The potential impact of PDI in PD brain is evidenced by experiments suggesting that PDI plays a role in the folding of both synphilin-1 and alpha-synuclein $[25,32]$, two proteins closely linked to PD. Other studies have also found links between PDI oxidation and PD [33, 34].

DJ- 1 activity is also altered by oxidative stress. DJ-1 is a $20 \mathrm{kDa}$ that has multiple putative activities as a protease and an antioxidant among others [35]. DJ-1 is strongly associated with PD because mutations in DJ-1 result in autosomal recessive early-onset form of Parkinson's disease. DJ-1 contains three cysteine residues that each has been evaluated in response to oxidative stress. Based upon multiple studies, it is clear that only Cys-106 oxidation is an important regulatory component for DJ-1 activity. For example, Waak and colleagues [36] found that the formation of a mixed disulfide, created under oxidizing conditions between DJ-1 and apoptosis signal-regulating kinase 1 (ASK1), contributes to DJ-1's neuroprotective effects. Such protective effects are predicted to be lost in cases that may occur with aging or exposure to oxidative toxins such as those used in animal models of PD including MPTP or rotenone.

Another example of a cysteine-containing enzyme that is modified in PD is parkin. Parkin is an ubiquitin E3 ligase that serves to ubiquitinate a series of proteins and contains multiple cysteines that are required for full activity. Mutation of parkin is responsible for early-onset autosomal recessive juvenile Parkinsonism. Several studies have convincingly demonstrated that parkin is S-nitrosylated in cases of PD as well as in model systems [37, 38]. Such oxidation inhibits parkin's ubiquitin E3 ligase activity and therefore prevents proper ubiquitination of its substrates leading to accumulation of misfolded proteins. In addition, it also appears to be sensitive to covalent modification by dopamine itself [39].

Tyrosine hydroxylase (TH) is the initial and rate-limiting step in the biosynthesis of the dopamine (DA) and norepinephrine. This enzyme contains seven cysteines some of which have been found to be important for full TH activity. Kuhn and colleagues [40] found that 4-5 cysteines were modified by quinone derivatives of DOPA, dopamine, and $\mathrm{N}$-acetyldopamine that were prevented by various thiolreducing agents. Further, they found that such oxidations resulted in inhibition of $\mathrm{TH}$ enzymatic activity. Other evidence of TH redox sensitivity comes from Sadidi et al., [41] who found that peroxnitrite and nitrogen dioxide both inhibited $\mathrm{TH}$ through nitration of cysteines or through Sthiolation in the presence of GSH or cysteine. Additional discussion of redox regulation of $\mathrm{TH}$ can be found in a recent, excellent review [42].

4.3. Amyotrophic Lateral Sclerosis. More commonly known as Lou Gehrig's disease, ALS is the most common degenerative disease of the motor neuron system that results in the death of motor neurons, causing muscle weakness and eventually death. Despite an annual incidence rate of oneto-two cases per 100,000, the etiology of the disease remains largely unknown [43]. Although multiple theories have been presented, research focusing on neurotoxicity has revealed excessive entry of glutamate into the neurons damages cell metabolism, resulting in pathologic changes [43]. It has been offered that ALS develops when vulnerable persons are exposed to a neurotoxin at times of strenuous physical activity [44].

4.3.1. Examples of Oxidized Enzymes in ALS. Mutations in $\mathrm{Cu} / \mathrm{Zn}$ superoxide dismutase gene (SOD1) are associated with familial amyotrophic lateral sclerosis. Recent work has found that oxidative modification of SOD1 results in the formation of an epitope consistent with misfolding of SOD1 that is observed in ALS [45]. Subsequently, Redler and colleagues [46] evaluated the effects of specific modification of Cys-111 on this conformation change and found oxidation of Cys-111 via glutathionylation (see Figure 1) resulted in the destabilization of the SOD1 dimer. This destabilization increases the potential for unfolding of the monomer and subsequent aggregation, leading to loss of SOD1 activity and promoting cell death.

\section{Other Cysteine-Dependent Enzymes Affected by Oxidative Stress}

Beyond cases clearly associated with specific disease pathology, there are other physiologically regulated or pathologically modified cysteine-dependent enzymes that are equally important to consider and have been suggested to play a role in neurodegeneration. For example, Janus kinase 2 (JAK2) 
TABLE 1: Examples of cysteine-dependent enzymes that use cysteine within their catalytic site within the various domains as delineated by the enzyme commission categories.

\begin{tabular}{|c|c|c|c|c|c|}
\hline Class 1 & Class 2 & Class 3 & Class 4 & Class 5 & Class 6 \\
\hline Oxidoreductases & Transferases & Hydrolases & Lyases & Isomerases & Ligases \\
\hline Protein-disulfide reductase & $\begin{array}{l}\text { Mercaptopyruvate } \\
\text { sulfurtransferase }\end{array}$ & PTEN & MerB* & $\begin{array}{l}\text { Protein disulfide } \\
\text { isomerase }\end{array}$ & Parkin \\
\hline Peroxiredoxin & Akt & Ubiquitinyl hydrolase 1 & LuxS* & GluRS* & \\
\hline $\begin{array}{l}\text { Glyceraldehyde-3-phosphate } \\
\text { dehydrogenase }\end{array}$ & Janus kinase 2 & Histone deacetylase & & & \\
\hline SOD1 & Sulfurtransferase & PTP1B & & & \\
\hline ALDH1L1 & $\begin{array}{l}\text { Epidermal growth factor } \\
\text { receptor }\end{array}$ & & & & \\
\hline Tyrosine hydroxylase & & & & & \\
\hline
\end{tabular}

is part of the JAK2/signal transducers and activators of Transcription pathway that plays a role in synaptic plasticity, cell proliferation, migration, and apoptosis. JAK2 contains a pair of cysteine residues (Cys866 and Cys917) that act as a redox-sensitive switch for its activity [47] and was shown to be inactivated by treatment of human BE (2)-C neuroblastoma cells with rotenone, a chemical used to model PD in animals [48].

Members of the caspase family are also found to be regulated by redox state. Caspases are involved in the initiation and execution of certain forms of programmed cell death and are therefore linked to multiple neurodegenerative conditions. Several studies have confirmed that members of this group can be oxidized at their active-site cysteine through S-nitrosylation, resulting in enzyme inhibition [4951]. However, there are other examples in which nitric oxide (NO) may activate these caspases [52]. Such discrepancies are likely due to duration and dose of $\mathrm{NO}$ as well as other indirect effects of NO on other activation mechanisms [53].

Phosphatase and tensin homolog (PTEN) dephosphorylates phosphatidylinositol $(3,4,5)$-trisphosphate $\left(\mathrm{PIP}_{3}\right)$ to phosphatidylinositol $(4,5)$-bisphosphate $\left(\mathrm{PIP}_{2}\right)$, serving to antagonize the kinase activity of phosphatidylinositide-3kinase. As part of this pathway that includes the Akt cascade, PTEN activity is relevant to apoptosis. Numajiri and colleagues [54] reported that S-nitrosylation of PTEN at Cys-83 inhibited PTEN activity resulting in increased AKT activity downstream, promoting cell survival. Interestingly, they also found that at higher levels of NO, AKT itself could be S-nitrosylated and therefore inhibited, resulting in a proapoptotic environment [54]. Finally, the active-site cysteine of PTEN, Cys124, has also been found to be oxidized in the presence of high concentrations of hydrogen peroxide [55].

Glyceraldehyde 3-phosphate dehydrogenase (GAPDH) is a glycolytic enzyme that also has other recently discovered roles including participating in apoptosis. Cumming and Schubert [56] showed that GAPDH is sensitive to oxidative stress in affected brain regions of $\mathrm{AD}$. They reported an increase in GAPDH intermolecular disulfide formation that was reversed by addition of the cysteine-specific reducing agent, dithiothreitol (DTT), that included the active-site Cys149. Treatment of cultured neuronal and neuronal-like cells with $\mathrm{A} \beta$ also resulted in GAPDH oxidation in addition to nuclear translocation and aggregation that may contribute to apoptosis [56]. As GAPDH's role is more fully elucidated, its oxidation is likely to be discovered to reach beyond what is presently known. See Table 1 for examples of cysteinedependent enzymes that have been found to be regulated by redox state within the various categories of enzymes.

\section{Cysteine-Dependent Enzymes and Their Link to Cancer}

Given the linkage between age and cancer, there are likely important connections between redox regulation of the enzymes associated with neurodegeneration discussed above and tumor formation. Indeed, there are many examples of cysteine-dependent enzymes playing important roles in the various aspects of cancer progression including the impact of cancer therapies on these enzymes. The following is a brief summary of examples of this overlap.

Wang and colleagues [57] found in their model using MCF-7 human breast cancer cells that became resistant to radiation, that $\operatorname{Prx} 2$ is upregulated and may be a contributing factor to resistance to radiation. They hypothesized that this may be due to the antioxidant function of Prx2, resulting in attenuating radiation-induced oxidative stress effects. Goplen and colleagues [58] found that PDI is highly expressed during glioma invasion and that treatment with bacitracin, or a monoclonal antibody to PDI, inhibited tumor migration and invasion. Calpain-2 has been shown to play a role in calcium-dependent glioblastoma invasion, but not migration, which may be related to calpain-2's function in invadopodial dynamics mediated by its regulation of matrix metalloproteinase 2 [59, 60]. DJ-1 appears to be upregulated in multiple forms of cancer and is considered a ras-dependent oncogene $[61,62]$. DJ-1 upregulation, as with Prx2, is likely due to its antioxidant properties and 
the protective effects it would convey upon tumor cells. Alterations of parkin, observed in multiple cancer types, with genetic or other causes of decreased parkin levels are linked to increased tumorigenesis. As recently reviewed, and epidemiological studies suggest, parkin, along with DJ-1 and other genetically linked proteins are under investigation with respect to increasing risk of melanoma in PD [6367]. SOD1, by virtue of its potent antioxidant activity, has been identified as a potential drug target to induce cell death in certain cancers. Somwar and colleagues [68], using a lung adenocarcinoma cell line, found that inhibition of SOD-1 led to increased apoptosis in these cells. Finally, Joshi and colleagues [69] treated mice with adriamycin, a chemotherapeutic agent, resulting in increased oxidation of Prx1, a cysteine-dependent peroxiredoxin, in brain.

From these data, two observations can be made. First, there are multiple cysteine-dependent enzymes that are sensitive to oxidative stress linked to tumor formation or migration. Second, the oxidizing effects of chemotherapeutic agents such as adriamycin should be considered when evaluating the potential effects of these compounds in terms of both their therapeutic and pathological effects.

\section{Summary}

Clearly, the redox regulation of cysteine-dependent enzymes is an important area of study. This is particularly evident in neurodegenerative conditions due to their strong association with increases in oxidative stress. Many of these same enzymes are also associated with tumorigenesis, invasion, or migration.

The selected enzymes for this paper appear not only sensitive to oxidation, but also key players in the underlying pathologies and in some cases, genetic causes of disease. It would appear that while nature has taken advantage of the reactivity of the sulfur group within cysteine to help regulate the response to oxidative stress, it also leaves these enzymes vulnerable to chronic conditions that promote prolonged exposure to an oxidizing environment. Thus, as our antioxidant defenses decline over time and cellular exposure to oxidizing conditions is increased, either through metabolic activity of the mitochondria, or by exposure to oxidizing environmental agents, this subset of cysteine-dependent enzymes become increasingly inhibited. Such inhibition is expected to contribute to and promote neurodegeneration, with variable effects on cancer. This paper has only highlighted some of the significant cysteinedependent enzymes that have been shown to be related to neurodegenerative diseases and not all of the tremendous efforts of the many researchers that have contributed have been referenced here.

\section{References}

[1] R. S. Pandya, L. L. J. Mao, E. W. Zhou et al., "Neuroprotection for amyotrophic lateral sclerosis: role of stem cells, growth factors, and gene therapy," Central Nervous System Agents in Medicinal Chemistry, vol. 12, no. 1, pp. 15-27, 2012.
[2] D. J. Surmeier, J. N. Guzman, J. Sanchez-Padilla, and J. A. Goldberg, "The origins of oxidant stress in parkinson's disease and therapeutic strategies," Antioxidants and Redox Signaling, vol. 14, no. 7, pp. 1289-1301, 2011.

[3] L. M. Sayre, M. A. Smith, and G. Perry, "Chemistry and biochemistry of oxidative stress in neurodegenerative disease," Current Medicinal Chemistry, vol. 8, no. 7, pp. 721-738, 2001.

[4] T. A. Clark, H. P. Lee, R. K. Rolston et al., "Oxidative stress and its implications for future treatments and management of Alzheimer disease," International Journal of Biomedical Science, vol. 6, no. 3, pp. 225-227, 2010.

[5] Y. Wang, J. Yang, and J. Yi, "Redox sensing by proteins: oxidative modifications on cysteines and the consequent events," Antioxidants and Redox Signaling, vol. 16, no. 7, pp. 649-657, 2012.

[6] V. I. Lushchak, "Adaptive response to oxidative stress: bacteria, fungi, plants and animals," Comparative Biochemistry and Physiology, vol. 153, no. 2, pp. 175-190, 2011.

[7] H. Ooe, S. M. M. Iguchi-Ariga, and H. Ariga, "Establishment of specific antibodies that recognize C106-oxidized DJ-1," Neuroscience Letters, vol. 404, no. 1-2, pp. 166-169, 2006.

[8] J. Hu, Z. Zhang, W.-J. Shen, A. Nomoto, and S. Azhar, "Differential roles of cysteine residues in the cellular trafficking, dimerization, and function of the high-density lipoprotein receptor, SR-BI," Biochemistry, vol. 50, no. 50, pp. 1086010875, 2011.

[9] P. Aracena-Parks, S. A. Goonasekera, C. P. Gilman, R. T. Dirksen, C. Hidalgo, and S. L. Hamilton, "Identification of cysteines involved in S-nitrosylation, S-glutathionylation, and oxidation to disulfides in ryanodine receptor type 1," The Journal of Biological Chemistry, vol. 281, no. 52, pp. 4035440368, 2006.

[10] S. Jordans, S. Jenko-Kokalj, N. M. Kühl et al., "Monitoring compartment-specific substrate cleavage by cathepsins B, K, $\mathrm{L}$, and $\mathrm{S}$ at physiological $\mathrm{pH}$ and redox conditions," $B M C$ Biochemistry, vol. 10, no. 1, article 23, 2009.

[11] K. Jomova and M. Valko, "Advances in metal-induced oxidative stress and human disease," Toxicology, vol. 283, no. 2-3, pp. 65-87, 2011.

[12] A. Spivey, "Rotenone and paraquat linked to Parkinson's Disease: human exposure study supports years of animal studies," Environmental Health Perspectives, vol. 119, no. 6, article A259, 2011.

[13] C. W. Olanow, "A rationale for monoamine oxidase inhibition as neuroprotective therapy for Parkinson's disease," Movement Disorders, vol. 8, no. 1, supplement, pp. S1-S7, 1993.

[14] H. M. Cochemé and M. P. Murphy, "Complex I is the major site of mitochondrial superoxide production by paraquat," The Journal of Biological Chemistry, vol. 283, no. 4, pp. 1786-1798, 2008.

[15] S. Pitkanen and B. H. Robinson, "Mitochondrial complex I deficiency leads to increased production of superoxide radicals and induction of superoxide dismutase," The Journal of Clinical Investigation, vol. 98, pp. 345-351, 1996.

[16] J. R. Treberg, C. L. Quinlan, and M. D. Brand, "Evidence for two sites of superoxide production by mitochondrial NADH-ubiquinone oxidoreductase (complex I)," The Journal of Biological Chemistry, vol. 286, no. 31, pp. 27103-27110, 2011.

[17] A. Nunomura, G. Perry, G. Aliev et al., "Oxidative damage is the earliest event in Alzheimer disease," Journal of $\mathrm{Neu}$ ropathology and Experimental Neurology, vol. 60, no. 8, pp. 759-767, 2001. 
[18] S. K. Garg, V. Vitvitsky, R. Albin, and R. Banerjee, "Astrocytic redox remodeling by amyloid beta peptide," Antioxidants and Redox Signaling, vol. 14, no. 12, pp. 2385-2397, 2011.

[19] M. A. Ansari and S. W. Scheff, "Oxidative stress in the progression of alzheimer disease in the frontal cortex," Journal of Neuropathology and Experimental Neurology, vol. 69, no. 2, pp. 155-167, 2010.

[20] A. Hall, P. A. Karplus, and L. B. Poole, "Typical 2-Cys peroxiredoxins-structures, mechanisms and functions," FEBS Journal, vol. 276, no. 9, pp. 2469-2477, 2009.

[21] T. T. Reed, W. M. Pierce Jr., D. M. Turner, W. R. Markesbery, and D. Allan Butterfield, "Proteomic identification of nitrated brain proteins in early Alzheimer's disease inferior parietal lobule," Journal of Cellular and Molecular Medicine, vol. 13, no. 8, pp. 2019-2029, 2009.

[22] R. C. Cumming, R. Dargusch, W. H. Fischer, and D. Schubert, "Increase in expression levels and resistance to sulfhydryl oxidation of peroxiredoxin isoforms in amyloid $\beta$-resistant nerve cells," The Journal of Biological Chemistry, vol. 282, no. 42, pp. 30523-30534, 2007.

[23] J. Fang, T. Nakamura, D. H. Cho, Z. Gu, and S. A. Lipton, "S-nitrosylation of peroxiredoxin 2 promotes oxidative stressinduced neuronal cell death in Parkinson's disease," Proceedings of the National Academy of Sciences of the United States of America, vol. 104, no. 47, pp. 18742-18747, 2007.

[24] Y. Honjo, H. Ito, T. Horibe, R. Takahashi, and K. Kawakami, "Protein disulfide isomerase-immunopositive inclusions in patients with Alzheimer disease," Brain Research, vol. 1349, pp. 90-96, 2010.

[25] T. Uehara, T. Nakamura, D. Yao et al., "S-Nitrosylated proteindisulphide isomerase links protein misfolding to neurodegeneration," Nature, vol. 441, no. 7092, pp. 513-517, 2006.

[26] D. E. Goll, V. F. Thompson, H. Li, W. Wei, and J. Cong, "The calpain system," Physiological Reviews, vol. 83, no. 3, pp. 731801, 2003.

[27] S. J. Storr, N. O. Carragher, M. C. Frame, T. Parr, and S. G. Martin, "The calpain system and cancer," Nature Reviews Cancer, vol. 11, no. 5, pp. 364-374, 2011.

[28] R. P. Guttmann, J. S. Elce, P. D. Bell, J. Clay Isbell, and G. V. W. Johnson, "'Oxidation inhibits substrate proteolysis by calpain I but not autolysis," The Journal of Biological Chemistry, vol. 272, no. 3, pp. 2005-2012, 1997.

[29] R. P. Guttmann and G. V. W. Johnson, "Oxidative stress inhibits calpain activity in situ," The Journal of Biological Chemistry, vol. 273, no. 21, pp. 13331-13338, 1998.

[30] J. L. Marcum, J. K. Mathenia, R. Chan, and R. P. Guttmann, "Oxidation of thiol-proteases in the hippocampus of Alzheimer's disease," Biochemical and Biophysical Research Communications, vol. 334, no. 2, pp. 342-348, 2005.

[31] S. Lesage and A. Brice, "Parkinson's disease: from monogenic forms to genetic susceptibility factors," Human Molecular Genetics, vol. 18, no. 1, pp. R48-R59, 2009.

[32] H. Cheng, L. Wang, and C. C. Wang, "Domain a of protein disulfide isomerase plays key role in inhibiting $\alpha$-synuclein fibril formation," Cell Stress and Chaperones, vol. 15, no. 4, pp. 415-421, 2010.

[33] K. J. Conn, W. Gao, A. McKee et al., "Identification of the protein disulfide isomerase family member PDIp in experimental Parkinson's disease and Lewy body pathology," Brain Research, vol. 1022, no. 1-2, pp. 164-172, 2004.

[34] R. Pal, M. Miranda, and M. Narayan, "Nitrosative stressinduced Parkinsonian Lewy-like aggregates prevented through polyphenolic phytochemical analog intervention," Biochemical and Biophysical Research Communications, vol. 404, no. 1, pp. 324-329, 2011.

[35] M. A. Wilson, "The role of cysteine oxidation in DJ-1 function and dysfunction," Antioxidants and Redox Signaling, vol. 15, no. 1, pp. 111-122, 2011

[36] J. Waak, S. S. Weber, K. Görner et al., "Oxidizable residues mediating protein stability and cytoprotective interaction of DJ-1 with apoptosis signal-regulating kinase 1," The Journal of Biological Chemistry, vol. 284, no. 21, pp. 14245-14257, 2009.

[37] F. Meng, D. Yao, Y. Shi et al., "Oxidation of the cysteine-rich regions of parkin perturbs its E3 ligase activity and contributes to protein aggregation," Molecular Neurodegeneration, vol. 6, no. 1 , article $34,2011$.

[38] E. S. P. Wong, J. M. M. Tan, C. Wang et al., "Relative sensitivity of parkin and other cysteine-containing enzymes to stress-induced solubility alterations," The Journal of Biological Chemistry, vol. 282, no. 16, pp. 12310-12318, 2007.

[39] J. R. Kim, K. S. Kwon, H. W. Yoon, S. R. Lee, and S. G. Rhee, "Oxidation of proteinaceous cysteine residues by dopaminederived $\mathrm{H}_{2} \mathrm{O}_{2}$ in PC12 cells," Archives of Biochemistry and Biophysics, vol. 397, no. 2, pp. 414-423, 2002.

[40] D. M. Kuhn, R. E. Arthur Jr., D. M. Thomas, and L. A. Elferink, "Tyrosine hydroxylase is inactivated by catechol-quinones and converted to a redox-cycling quinoprotein: possible relevance to Parkinson's disease," Journal of Neurochemistry, vol. 73, no. 3, pp. 1309-1317, 1999.

[41] M. Sadidi, T. J. Geddes, and D. M. Kuhn, "S-thiolation of tyrosine hydroxylase by reactive nitrogen species in the presence of cysteine or glutathione," Antioxidants and Redox Signaling, vol. 7, no. 7-8, pp. 863-869, 2005.

[42] G. Di Giovanni, M. Pessia, and R. Di Maio, "Redox sensitivity of tyrosine hydroxylase activity and expression in dopaminergic dysfunction," CNS \& Neurological Disorders: Drug Targets, vol. 11, no. 4, pp. 419-429, 2012.

[43] A. D. Walling, "Amyotrophic lateral sclerosis: lou Gehrig's disease," American Family Physician, vol. 59, no. 6, pp. 14891496, 1999.

[44] W. T. Longstreth, L. M. Nelson, T. D. Koepsell, and G. Van Belle, "Hypotheses to explain the association between vigorous physical activity and amyotrophic lateral sclerosis," Medical Hypotheses, vol. 34, no. 2, pp. 144-148, 1991.

[45] D. A. Bosco, G. Morfini, N. M. Karabacak et al., "Wild-type and mutant SOD1 share an aberrant conformation and a common pathogenic pathway in ALS," Nature Neuroscience, vol. 13, no. 11, pp. 1396-1403, 2010.

[46] R. L. Redler, K. C. Wilcox, E. A. Proctor, L. Fee, M. Caplow, and N. V. Dokholyan, "Glutathionylation at Cys-111 induces dissociation of wild type and FALS mutant SOD1 dimers," Biochemistry, vol. 50, no. 32, pp. 7057-7066, 2011.

[47] J. K. Smith, C. N. Patil, S. Patlolla, B. W. Gunter, G. W. Booz, and R. J. Duhé, "Identification of a redox-sensitive switch within the JAK2 catalytic domain," Free Radical Biology and Medicine, vol. 52, no. 6, pp. 1101-1110, 2012.

[48] R. K. Monroe and S. W. Halvorsen, "Environmental toxicants inhibit neuronal Jak tyrosine kinase by mitochondrial disruption," NeuroToxicology, vol. 30, no. 4, pp. 589-598, 2009.

[49] J. Haendeler, U. Weiland, A. M. Zeiher, and S. Dimmeler, "Effects of redox-related congeners of NO on apoptosis and caspase-3 activity," Nitric Oxide, vol. 1, no. 4, pp. 282-293, 1997.

[50] L. Liu and J. S. Stamler, "NO: an inhibitor of cell death," Cell Death and Differentiation, vol. 6, no. 10, pp. 937-942, 1999. 
[51] G. Melino, M. V. Catani, M. Corazzari, P. Guerrieri, and F. Bernassola, "Nitric oxide can inhibit apoptosis or switch it into necrosis," Cellular and Molecular Life Sciences, vol. 57, no. 4, pp. 612-622, 2000.

[52] A. Adamczyk, A. Kaźmierczak, G. A. Czapski, and J. B. Strosznajder, " $\alpha$-Synuclein induced cell death in mouse hippocampal (HT22) cells is mediated by nitric oxide-dependent activation of caspase-3," FEBS Letters, vol. 584, no. 15, pp. 3504-3508, 2010.

[53] Y. C. Kang, P. K. Kim, B. M. Choi et al., "Regulation of programmed cell death in neuronal cells by nitric oxide," In Vivo, vol. 18, no. 3, pp. 367-376, 2004.

[54] N. Numajiri, K. Takasawa, T. Nishiya et al., "On-off system for PI3-kinase-Akt signaling through S-nitrosylation of phosphatase with sequence homology to tensin (PTEN)," Proceedings of the National Academy of Sciences of the United States of America, vol. 108, no. 25, pp. 10349-10354, 2011.

[55] S. R. Lee, K. S. Yang, J. Kwon, C. Lee, W. Jeong, and S. G. Rhee, "Reversible inactivation of the tumor suppressor PTEN by $\mathrm{H}_{2} \mathrm{O}_{2}$," The Journal of Biological Chemistry, vol. 277, no. 23, pp. 20336-20342, 2002.

[56] R. C. Cumming and D. Schubert, "Amyloid- $\beta$ induces disulfide bonding and aggregation of GAPDH in Alzheimer's disease," FASEB Journal, vol. 19, no. 14, pp. 2060-2062, 2005.

[57] T. Wang, D. Tamae, T. LeBon, J. E. Shively, Y. Yen, and J. J. $\mathrm{Li}$, "The role of peroxiredoxin II in radiation-resistant MCF-7 breast cancer cells," Cancer Research, vol. 65, no. 22, pp. 1033810346, 2005.

[58] D. Goplen, J. Wang, P. Ø. Enger et al., "Protein disulfide isomerase expression is related to the invasive properties of malignant glioma," Cancer Research, vol. 66, no. 20, pp. 98959902, 2006.

[59] H. S. Jang, S. Lal, and J. A. Greenwood, "Calpain 2 is required for glioblastoma cell invasion: regulation of matrix metalloproteinase 2," Neurochemical Research, vol. 35, no. 11, pp. 1796-1804, 2010.

[60] S. Lal, J. La Du, R. L. Tanguay, and J. A. Greenwood, "Calpain 2 is required for the invasion of glioblastoma cells in the zebrafish brain microenvironment," Journal of Neuroscience Research, vol. 90, pp. 769-781, 2012.

[61] C. M. Clements, R. S. McNally, B. J. Conti, T. W. Mak, and J. P. Y. Ting, "DJ-1, a cancer- and Parkinson's diseaseassociated protein, stabilizes the antioxidant transcriptional master regulator Nrf2," Proceedings of the National Academy of Sciences of the United States of America, vol. 103, no. 41, pp. 15091-15096, 2006.

[62] R. S. McNally, B. K. Davis, C. M. Clements, M. A. AccavittiLoper, T. W. Mak, and J. P. Y. Ting, "DJ-1 enhances cell survival through the binding of Cezanne, a negative regulator of NF$\kappa \mathrm{B}$," The Journal of Biological Chemistry, vol. 286, no. 6, pp. 4098-4106, 2011.

[63] J. M. Bertoni, J. P. Arlette, H. H. Fernandez et al., "Increased melanoma risk in Parkinson disease: a prospective clinicopathological study," Archives of Neurology, vol. 67, no. 3, pp. 347-352, 2010.

[64] M. Fujiwara, H. Marusawa, H. Q. Wang et al., "Parkin as a tumor suppressor gene for hepatocellular carcinoma," Oncogene, vol. 27, no. 46, pp. 6002-6011, 2008.

[65] G. Poulogiannis, R. E. McIntyre, M. Dimitriadi et al., "PARK2 deletions occur frequently in sporadic colorectal cancer and accelerate adenoma development in Apc mutant mice," Proceedings of the National Academy of Sciences of the United States of America, vol. 107, no. 34, pp. 15145-15150, 2010.
[66] Y. C. Kim, H. Kitaura, S. M. M. Iguchi-Ariga, and H. Ariga, "DJ-1, an oncogene and causative gene for familial Parkinson's disease, is essential for SV40 transformation in mouse fibroblasts through up-regulation of c-Myc," FEBS Letters, vol. 584, no. 18, pp. 3891-3895, 2010.

[67] H. Ren, K. Fu, C. Mu, B. Li, D. Wang, and G. Wang, "DJ-1, a cancer and Parkinson's disease associated protein, regulates autophagy through JNK pathway in cancer cells," Cancer Letters, vol. 297, no. 1, pp. 101-108, 2010.

[68] R. Somwar, H. Erdjument-Bromage, E. Larsson et al., "Superoxide dismutase 1 (SOD1) is a target for a small molecule identified in a screen for inhibitors of the growth of lung adenocarcinoma cell lines," Proceedings of the National Academy of Sciences of the United States of America, vol. 108, pp. 1637516380, 2011.

[69] G. Joshi, C. D. Aluise, M. P. Cole et al., "Alterations in brain antioxidant enzymes and redox proteomic identification of oxidized brain proteins induced by the anti-cancer drug adriamycin: implications for oxidative stress-mediated chemobrain," Neuroscience, vol. 166, no. 3, pp. 796-807, 2010. 

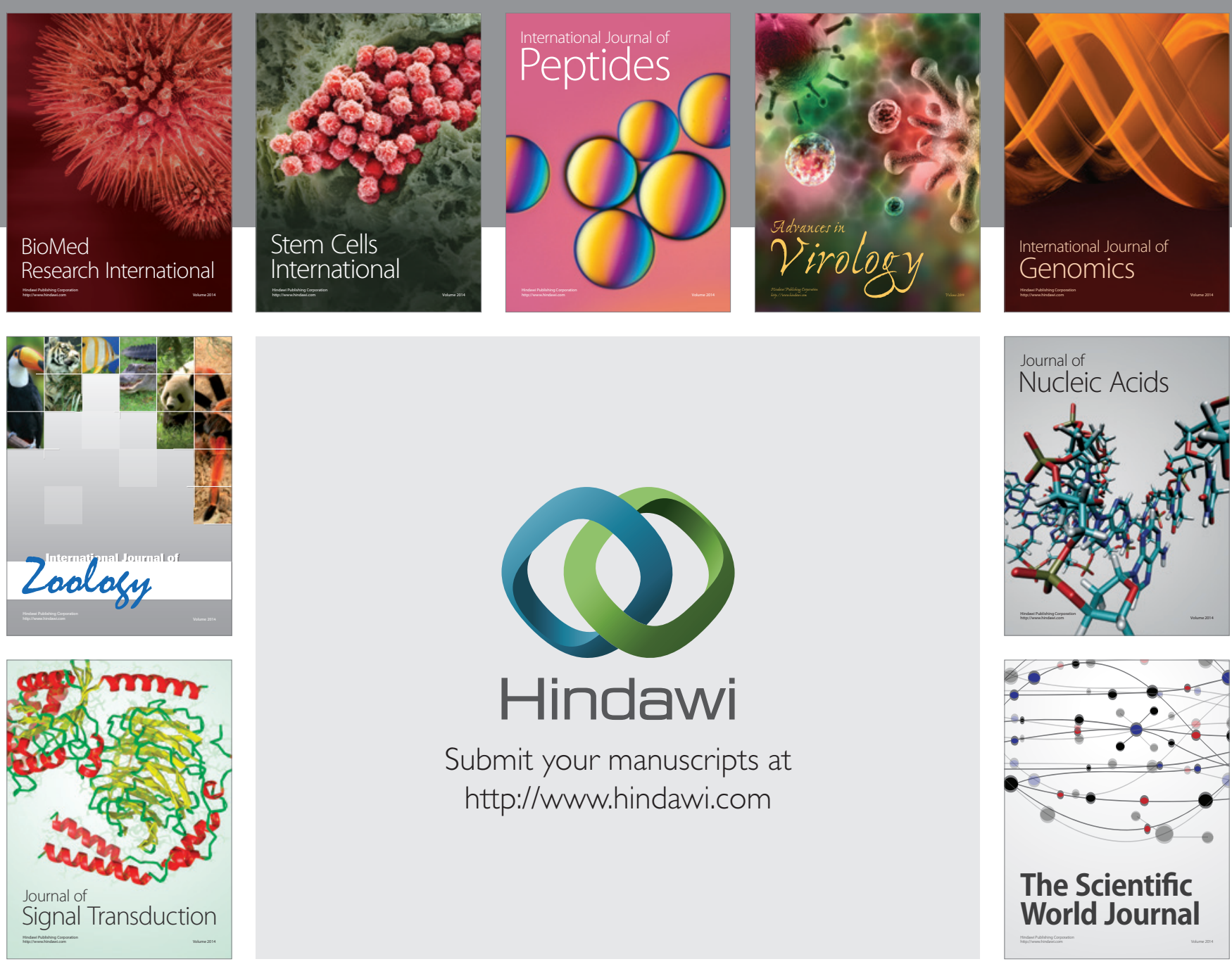

Submit your manuscripts at

http://www.hindawi.com
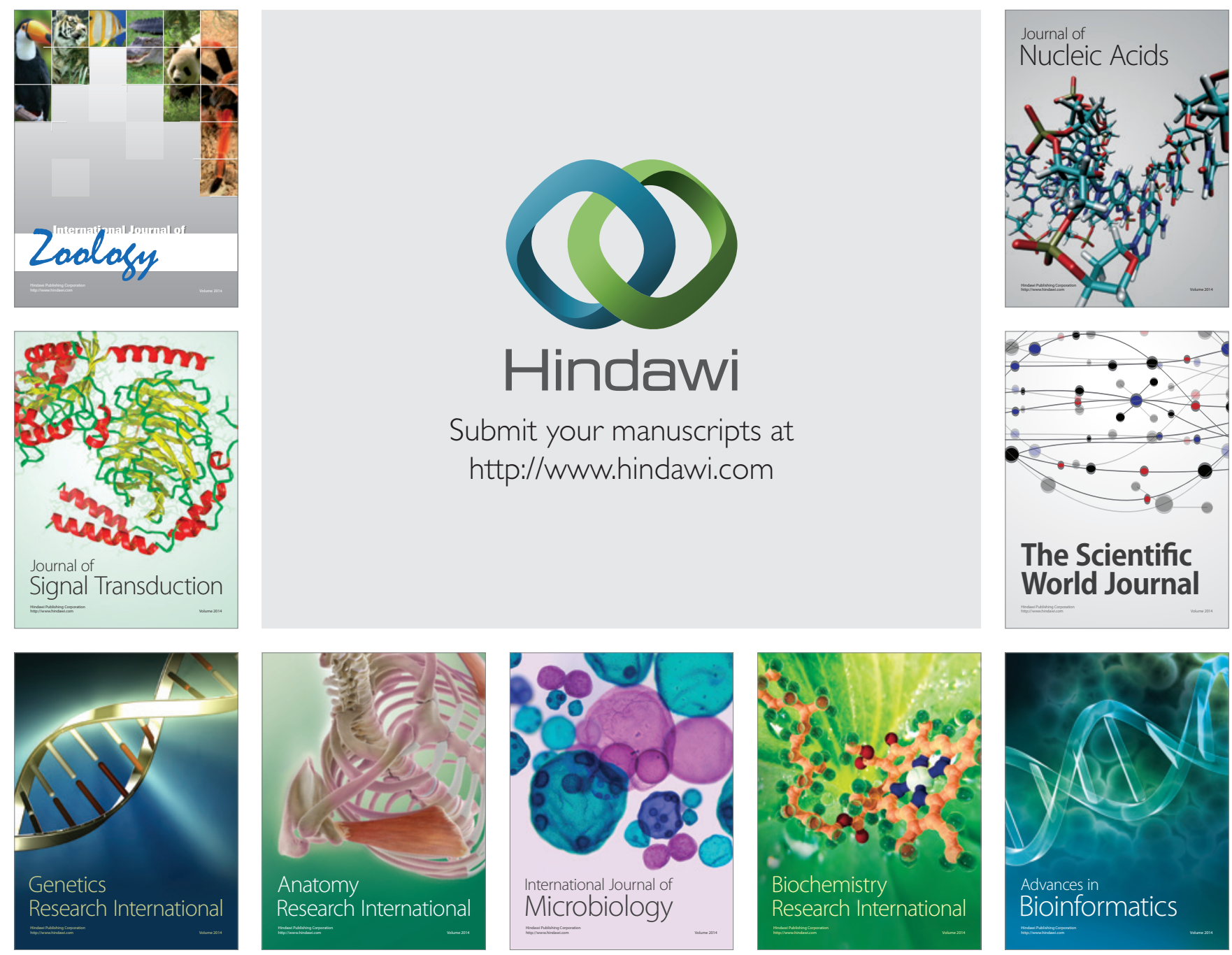

The Scientific World Journal
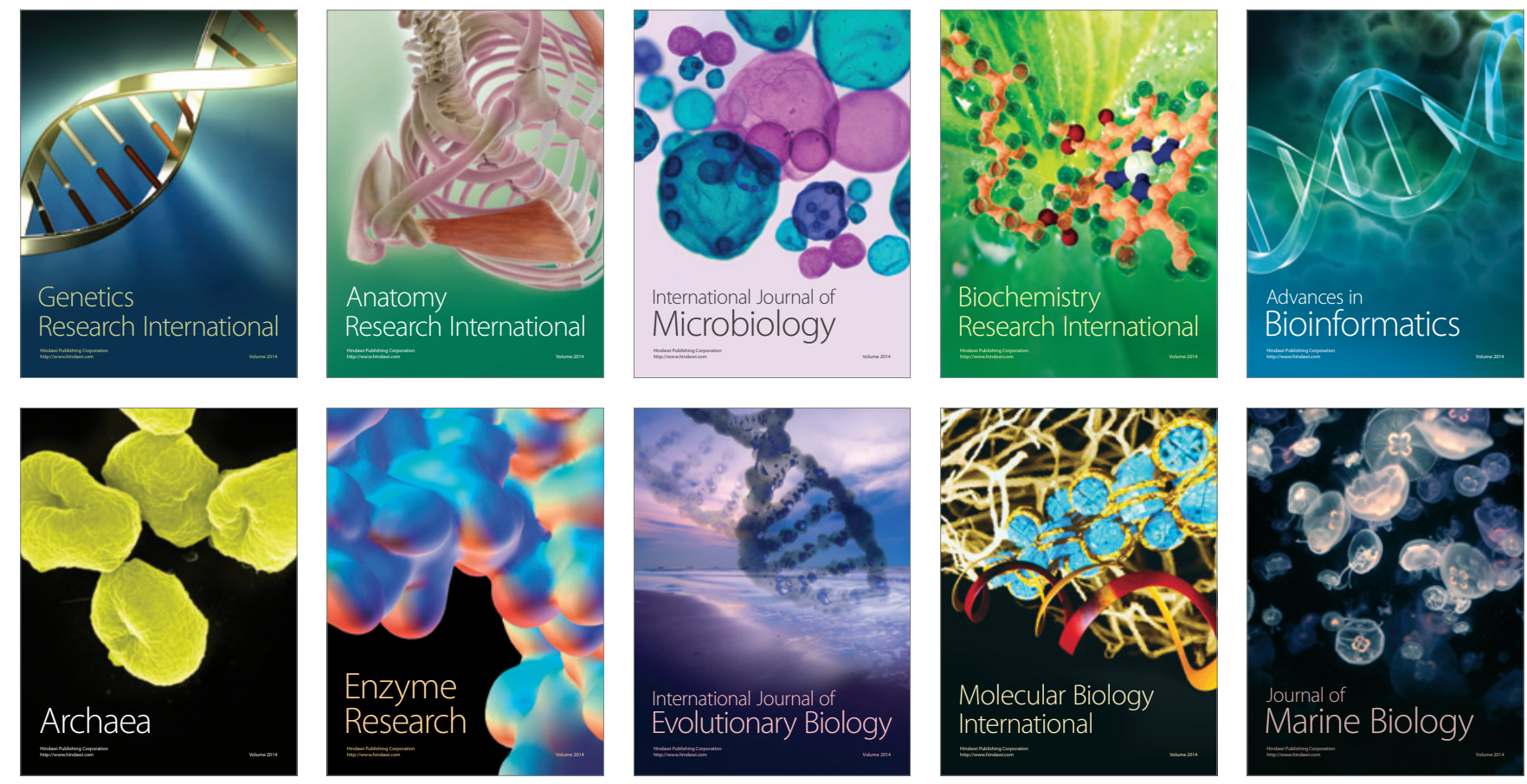